\title{
Clinicopathological and genetic features of Chinese hereditary nonpolyposis colorectal cancer (HNPCC)
}

\author{
Fangqi Liu $\cdot$ Li Yang $\cdot$ Xiaoyan Zhou $\cdot$ \\ Weiqi Sheng $\cdot$ Sanjun Cai $\cdot$ Lei Liu • \\ Peng Nan $\cdot$ Ye Xu
}

Received: 10 August 2014/ Accepted: 2 September 2014/Published online: 13 September 2014

(C) The Author(s) 2014. This article is published with open access at Springerlink.com

\begin{abstract}
The aim of this study was to investigate the clinical value of different criteria and to understand the relationship between genotype and phenotype in Chinese hereditary nonpolyposis colorectal cancer (HNPCC). A total of 116 unrelated probands of suspected HNPCC families from the Fudan Colorectal Registry were studied. A total of 32, 28, and 56 families fulfilled the Amsterdam criteria, the Fudan criteria and the revised Bethesda guideline, respectively. Direct DNA sequencing of all exons of hMSH2 and hMLH1 genes were performed on all 116 samples. Mutations and clinicopathological features were compared between the groups. Thirty-two pathological germline mutations were identified. Out of 32 mutations, 16 were located at hMLH1 and 16 at hMSH2. The
\end{abstract}

Fangqi Liu and Li Yang have contributed equally to this work.

F. Liu $\cdot$ L. Yang $\cdot$ S. Cai $\cdot$ Y. Xu $(\bowtie)$

Department of Colorectal Surgery, Fudan University Shanghai

Cancer Center, Shanghai 200032, China

e-mail: xuye021@163.com; xu_shirley021@163.com

F. Liu $\cdot$ L. Yang $\cdot$ X. Zhou $\cdot$ W. Sheng $\cdot$ S. Cai $\cdot$ Y. Xu

Department of Oncology, Shanghai Medical College, Fudan

University, Shanghai 200032, China

X. Zhou $\cdot$ W. Sheng

Department of Pathology, Fudan University Shanghai Cancer

Center, Shanghai 200032, China

L. Liu

Shanghai Medical College of Fudan University,

Shanghai 200032, China

P. Nan

Ministry of Education Key Laboratory for Biodiversity Science and Ecological Engineering, School of Life Sciences, Fudan University, Shanghai 200433, China

e-mail: nanpeng@fudan.edu.cn sensitivity of Amsterdam criteria was $50 \%$, specificity was $81 \%$, and Youden's index was $31 \%$. The sensitivity of Fudan criteria was $75 \%$, specificity was $58 \%$, and Youden's index was $33 \%$. Among all the 32 families with mutations, families with hMSH2 mutation had a higher ratio of synchronous and metachronous colon cancers than families with hMLH1 mutation (33 vs. $6 \%, P=0.04$ ). Patients with hMSH2 mutation more frequently harbour synchronous and metachronous colon cancers. Fudan criteria had a little higher sensitivity and accuracy than Amsterdam criteria for identification of Chinese HNPCC.

Keywords HNPCC - Clinicopathological features . MLH1/MSH2 mutations · Clinical criteria

\section{Introduction}

Hereditary nonpolyposis colorectal cancer (HNPCC), also known as Lynch Syndrome, is an autosomal-dominant syndrome accounting for 3-5\% of colorectal cancer cases $[1,2]$. Germline mutations resulting in HNPCC have been found in six mismatch repair (MMR) genes (hMLH1, hMSH2, hMSH3, hMSH6, hPMS1, and hPMS2) [3-6]. Most $(90 \%)$ of the mutations locate in two MMR genes, MLH1 (50\%) and MSH2 (40\%) [7]. Lynch viewed HNPCC as a syndrome characterized by an autosomaldominant pattern of inheritance, early onset of malignancy with a predilection for the proximal colon, multiple CRCs, the absence of premonitory lesions (e.g. adenomas), and the occurrence of cancer in certain extracolonic sites, notably endometrium and ovary $[2,8]$.

Traditionally, screening for HNPCC has relied on examination of family history and other clinicopathological criteria, such as the Amsterdam criteria [9]. Adding the 
Bethesda guidelines to the diagnostic process allowed the identification of more individuals likely to have HNPCC $[10,11]$. However, the Bethesda guidelines are not highly specific. Studies of families meeting the Amsterdam criteria have identified germline MSH2 and MLH1 mutations with a relatively high sensitivity $(\sim 60 \%)$ and specificity ( $\sim 70 \%$ ). In contrast, germline MSH2 and MLH1 mutations were found with a higher sensitivity ( $94 \%)$ and a lower specificity $(\sim 30 \%)$ when families meeting the Bethesda criteria were studied [12]. Compared with these two criteria, one of the differences is extracolonic cancers types. Stomach, ovary and brain cancers were not included in the Amsterdam criteria. The extracolonic cancers of AC II only included endometrial cancer, small intestinal cancer and ureter or renal pelvis cancer, because gastric cancer was only frequently reported in Asian HNPCC families, and it is uncommon and is observed mainly in patients from older generations in Western countries. According to previous studies on HNPCC, there were many differences between the Western and the Eastern countries [13]. For example, a predominance of distal tumours was reported in Asian patients with HNPCC [14], and our previous studies showed that the primary extracolonic tumours in stomach were more frequent [15]. Gastric cancer is the second most common extracolonic malignancy in HNPCC [16], and in some Asian populations, it is even the most common extracolonic cancer [17, 18]. According to the clinical features of Asian population, Fudan University Shanghai Cancer Center suggested include gastric cancer in Amsterdam criteria II and regarded this changed standard as "Fudan criteria", which was first mentioned in 2004 China Tumour Clinical Yearbook.

The golden standard for diagnostic of HNPCC is germline mutation in MMR genes. At present, direct sequencing is the key procedure in recognizing MMR genes defects [19]. Molecular genetic heterogeneity must be considered when assessing the Lynch syndrome cancer phenotype. It is important to appreciate that there are important differences between the different forms of Lynch syndrome. For example, Lynch syndrome-MLH1 type appears to be associated with a deficit of extracolonic cancers (such as endometrial cancers) and an excess of CRCs, when compared with Lynch syndrome-MSH2 type, which is prominently associated with extracolonic cancers. In patients with endometrial cancer, MMR gene mutations are most prevalent in the MSH2 gene (5.2-7.0\%). On the other hand, Lynch syndrome-MSH6 type is associated with later onset CRCs and a greater number of endometrial carcinomas. Lynch syndrome-PMS2 type appears to have a later age of onset of $\mathrm{CRC}$, but we do not know enough about these families to provide a complete description of how they differ from the phenotypes mentioned above [20,
21]. So far, only small genotype-phenotype studies have been performed to clarify the risks for patients specific to the MMR gene affected. Prediction of clinical risks and outcomes associated with each gene has not been possible in a reliable manner.

The aim of this study is to investigate the clinical value of different criteria and to understand the relationship between genotype and phenotype in Chinese HNPCC.

\section{Materials and methods}

\section{Patients}

A total of 116 families with suspected HNPCC were studied from 1994 to 2008 in Shanghai Cancer Hospital of Fudan University. All were native Chinese without immigrant ancestors. Thirty-two families fulfilled Amsterdam II criteria and were classified as AC group. Among the rest 84 families, there were 28 who met the Fudan criteria were classified as FD group.

Information of the probands and their relatives, i.e. age of onset, site of the CRCs, operative notes, pathological report, synchronous or metachronous colorectal cancers, extracolonic cancers, were documented. Available information of family history was documented for each family in a fourth-generation pedigree.

\section{Extraction of DNA from blood}

Blood samples were taken from 116 probands. Genomic DNA was isolated from peripheral blood lymphocytes according to the manufacturer's instructions (TIANGEN BIOTECH, BEIJING). The extracted genomic DNA was stored at $-20{ }^{\circ} \mathrm{C}$ for further analysis.

\section{Mutation of MLH1 and MSH2 genes}

Exons 1-19 of MLH1 and exons 1-16 of MSH2, including the splice-site junctions, were amplified by polymerase chain reaction (TaKaKa Biotechnology, Dalian). The PCR products were purified by $1.5 \%$ agarose gel electrophoresis, following the manufacturer's instructions (TIANGEN BIOTECH, BEIJING). All the purified products were sequenced by Shanghai Sunny Biotechnology Co., Ltd with 3730XL of ABI. Each mutation was amplified for both the sense and antisense strand, and then, the experiment was repeated at least once. If the mutation was exactly the same through at least three repeated experiments, the mutation was confirmed. By comparing with the reference sequences, missense and frameshift mutations were identified. 
Table 1 AC group versus FD group (CRC; colorectal cancer)

\begin{tabular}{llll}
\hline & Amsterdam criteria 32 & Fudan criteria & $P$ value \\
\hline Families & & 28 & \\
Related cancer patients/related cancers & $139 / 191$ & $112 / 120$ & \\
CRC cancer patients/CRC cancers & $114 / 155$ & $50 / 53$ \\
Male/female & $82 / 56$ & $63 / 49$ & \\
Age of first CRC & 36.5 & 46.6 & 0.530 \\
Age of first related cancers & 35.4 & 41.7 & 0.976 \\
Right colon cancer & $75 / 155(48.4 \%)$ & $24 / 53(45.3 \%)$ & 0.207 \\
Synchronous or metachronous colorectal cancers & $26 / 114(22.8 \%)$ & $3 / 50(6 \%)$ & 0.255 \\
Synchronous or metachronous related cancers & $30 / 139(21.6 \%)$ & $7 / 112(6 \%)$ & 0.001 \\
Extracolonic cancers & $35 / 191(18.3 \%)$ & $67 / 120(55.8 \%)$ & 0.000 \\
Mucinous adenocarcinoma & $9 / 27(33.3 \%)$ & $4 / 26(15.4 \%)$ & 0.868 \\
Stage III/IV CRCs & $8 / 27(29.6 \%)$ & $6 / 26(23.1 \%)$ & 0.550 \\
\hline
\end{tabular}

Definition of pathogenic mutations

Sequence variants that would obviously impact the function of MLH1 or MSH2 proteins, such as nonsense and frameshift mutations, were considered pathogenic. Besides, the mutations at highly conserved splice sites were also considered pathogenic. All the missense mutations were assessed for pathogenicity by searching against the InSiGHT database (www.insight-group.org), MMR Gene Unclassified Variants Database (www.mmruv.info), Mismatch Repair Genes Variants Database (www.med.mun.ca/ MMRvariants), and the Human Gene Mutation Database (www.hgmd.cf.ac.uk/ac). If the missense mutations affected the promoter site, or have been reported as pathogenic mutations in other studies, they were also considered pathogenic. Those missense mutations whose pathogenicity could not be confirmed were classified as uncertain.

\section{Statistical analysis}

Differences in the frequency distributions of categorical factors, sex, age at diagnosis, tumour location and pathology, were compared between Amsterdam I/II criteria and Fudan criteria using partitions of the chi-square test. All the statistical analysis was carried out using SPSS statistical software (version 15.0). A $P$ value of $<0.05$ was considered statistically significant.

\section{Results}

\section{MLH1 and MSH2 mutations}

Thirty-two pathological germline mutations were identified. Sixteen mutations were located at hMLH1, and 16 were located at hMSH2. There was a wide spectrum of mutation type, including 14 missense, 7 nonsense, 4 splice site, 2 frame insertion or deletion, and 5 frame shift mutations. Among the 16 mutations of hMLH1, exon12 and exon 15 both occupied three mutations. Among the 16 mutations of hMLH2, exon7 had six.

Amsterdam criteria (AC group) and Fudan criteria (FD group)

Compared the clinicopathological features between families belonging to these two groups, only two significant differences were discovered. More synchronous and metachronous multiple relative tumours (21.6 vs. $6 \%$, $P=0.001)$ were found in AC group, while more extracolorectal tumours $(55.8$ vs. $18.3 \%, P=0.000)$ were found in FD group. Other features such as earlier age of onset for all colorectal cancers and all tumours, the ratio of proximal colonic cancers, the ratio of synchronous and metachronous multiple colon cancers, the ratio of mucinous carcinoma and the ratio of stage III/IV colon cancer were similar (Table 1). Sixteen mutations were detected in 32 families of AC group. Using Fudan criteria, 24 mutations were detected in 60 families. The sensitivity of Amsterdam Criteria was $50 \%$, specificity was $81 \%$, and Youden's index was $31 \%$. The sensitivity of Fudan criteria was $75 \%$, specificity was $58 \%$, and Youden's index was $33 \%$.

Relationship between genotype and phenotype

In families of $\mathrm{AC}$ group, we divided them into group $\mathrm{A}$ (with MMR mutation) and group B (without MMR mutation); there were no significant clinicopathological differences between these two groups (Table 2).

Among all the 32 families with mutations, those with hMSH2 mutation had a higher ratio of synchronous and metachronous colon cancers than families with hMLH1 mutation (33 vs. $6 \%, P=0.04$ ) (Table 3). 
Table 2 Mutation versus nonmutation in Amsterdam criteria group

Table 3 MLH1 mutation versus MSH2 mutation

\begin{tabular}{llll}
\hline & Mutation & Non-mutation & $P$ value \\
\hline Families & 16 & 16 & \\
Related cancer patients/related cancers & $73 / 107$ & $66 / 84$ & \\
CRC cancer patients/CRC cancers & $60 / 86$ & $54 / 69$ & \\
Male/female & $41 / 31$ & $41 / 25$ & 0.738 \\
Age of first CRC & 36.9 & 36.1 & 0.932 \\
Age of first related cancers & 34.8 & 36.1 & 0.054 \\
Right colon cancer & $47 / 86(54.7 \%)$ & $28 / 69(40.6 \%)$ & 0.530 \\
Synchronous or metachronous colorectal cancers & $16 / 60(26.7 \%)$ & $10 / 54(18.5 \%)$ & 0.172 \\
Synchronous or metachronous related cancers & $20 / 73(27.4 \%)$ & $10 / 66(15.2 \%)$ & 0.574 \\
Extracolonic cancers & $19 / 107(17.8 \%)$ & $16 / 66(24.2 \%)$ & $0.57 \%$ \\
Mucinous adenocarcinoma & $6 / 13(46.2 \%)$ & $3 / 14(21.4 \%)$ & 0.234 \\
Stage III/IV CRCs & $4 / 13(30.8 \%)$ & $4 / 14(28.6 \%)$ & 0.730 \\
\hline
\end{tabular}

\begin{tabular}{llll}
\hline & MLH1 & MSH2 & $P$ value \\
\hline Families & 16 & 16 & \\
Related cancer patients/related cancers & $66 / 86$ & $61 / 88$ & \\
CRC cancer patients/CRC cancers & $47 / 59$ & $45 / 65$ & \\
Male/female & $38 / 28$ & $36 / 24$ & \\
Age of first CRC & 43.4 & 37.9 & 0.166 \\
Age of first related cancers & 40.3 & 37.1 & 0.419 \\
Right colon cancer & $37 / 59(62.7 \%)$ & $34 / 65(52.3 \%)$ & 0.748 \\
Synchronous or metachronous colorectal cancers & $3 / 47(6 \%)$ & $15 / 45(33.3 \%)$ & 0.04 \\
Synchronous or metachronous related cancers & $14 / 66(21.2 \%)$ & $18 / 61(29.5 \%)$ & 0.433 \\
Extracolonic cancers & $25 / 86(29.1 \%)$ & $23 / 88(26.1 \%)$ & 0.860 \\
Mucinous adenocarcinoma & $3 / 15(20 \%)$ & $7 / 13(53.8 \%)$ & 0.127 \\
Stage III/IV CRCs & $6 / 15(40 \%)$ & $4 / 13(30.8 \%)$ & 0.502 \\
\hline
\end{tabular}

\section{Discussion}

Clinical features and clinical diagnostic criteria

Among the 116 probands we collected, more left colon cancers than the right were found (64.7 vs. $35.3 \%)$, especially the rectal cancers. MMR gene mutation is regarded as the gold standard of HNPCC. So, 32 probands with MLH1 or MSH2 positive mutation and their relatives need to be analyzed in detail. The results showed that within these 32 families, 124 CRC happened, 70 were left side, and 54 were right ( 56.5 vs. $43.5 \%$ ), similar to the data above. The reason may depend on the higher risk of rectal cancers in China, or this difference may exactly be the Asian HNPCC feature. Chew MH et al. [14] also mentioned this different in their report. A higher predominance of right-sided CRC as reported in most HNPCC [22, 23]. However, rectal cancers were far more frequent than previously reported [24]. Timm Goecke also reported rectal cancers were remarkably frequent in $281 \mathrm{MLH} 1 / \mathrm{MSH} 2$ mutation carriers in German [25]. This indicates the need of more attention to rectal cancers in HNPCC patients, not only in Asian countries but also in Western countries.

In these 32 probands with MLH1 or MSH2 positive mutation and their relatives, focusing on the extracolonic tumours, 48 related cancers were happened, most of which were gastric cancer and endometrial carcinoma, 12 each $(25 \%)$. And similar results were reported in Korea and Brazil [26, 27]. It was significantly different from most European and American HNPCC families whose most frequent extracolonic cancer was endometrial cancer [8, 28, 29]. But for Asian population, gastric cancer is one of the most frequently extracolonic cancer in the current HNPCC and suspected families [30]. Some researchers pointed out that poor living standard may be the reason to explain the high frequency of gastric cancer. The frequency of cancer in individual organs can vary substantially depending upon ethnic, racial, and geographic differences [31]. In our opinion, gastric cancer may play a particular role in HNPCC families. In fact, some studies from Europe 
and America also supported that gastric cancer was associated with HNPCC syndrome [27, 32]. According to a study by Watson and Lynch that was performed in America, the gastric cancer risk in the familial members of HNPCC was increased 4.1-fold over the general population [33]. Some studies also show the relative risk of gastric cancer in HNPCC mutation carriers compared with the general population has been reported to be higher by 4-19fold [34] in populations of the Western world and at least by twofold in endemic areas in Asia [17]. Aarnio et al. [35] calculated the lifetime risk of gastric cancer in mutation carriers of the HNPCC gene as $19 \%$ in the Finnish population. These studies all gastric cancer is the main related cancer in HNPCC family.

Back to our study, 32 probands fulfilled Amsterdam I/II criteria, $16(50 \%)$ have the MMR mutation. If using Fudan criteria, gastric cancer plused into the related cancer of Amsterdam II criteria, 60 probands were fulfilled, 24 of them $(40 \%)$ occupied MRR mutation. The sensitivity of Amsterdam Criteria was $50 \%$, specificity was $81 \%$, and Youden's index was $31 \%$. The sensitivity of the changed criteria (Fudan criteria) was $75 \%$, specificity was $58 \%$, and Youden's index was $33 \%$. Although the Amsterdam criteria have been extremely successful in achieving their original purpose of providing a common nomenclature for the HNPCC syndrome for research purposes, using these criteria in the clinical realm must be done with extreme caution. Their limited sensitivity for identifying families with MSH2 and MLH1 mutations make it inappropriate to use the Amsterdam criteria as the sole criteria in choosing which patients should undergo genetic testing. Sapna Syngal et al. [12] showed that the sensitivity of the Modified Amsterdam and Amsterdam II criteria were $72 \%$ (95\% CI 58-86) and $78 \%$ (95\% CI 64-92), respectively. Overall, the most sensitive criteria for identifying families with pathogenic mutations were the Bethesda criteria, with a sensitivity of $94 \%$ (95\% CI 88-100); the specificity of these criteria was $25 \%$ (95\% CI 14-36). Obviously, greater sensitivity of the Bethesda guidelines was achieved at the expense of decreased specificity. The Bethesda Guidelines were proposed to target who should undergo tumour MSI analysis. However, the use of MSI testing in clinical practice has some major practical obstacles: MSI testing as a routine commercial clinical laboratory test is not widely available, and tumour blocks are often difficult to obtain/analyse owing to logistical and technical difficulties [36]. In our country, many HNPCC patients were found in outpatient department and they had already received the operation in local hospital, so tumour tissues were hardly got because the patients were from all of the provinces. Based on these limitations of Bethesda Guidelines, Fudan criteria can still keep the standard not too board as Bethesda criteria. Another important sense is that with the same detection rate as Amsterdam I/II criteria, more HNPCC families could be detected by using Fudan criteria.

Further more, each feature of HNPCC between Amsterdam I/II criteria and Fudan criteria were compared. Include age of onset, sex, site of the colorectal cancer, synchronous or metachronous tumours rate, and mucinous carcinoma rate, there were no significant differences, except synchronous or metachronous tumours rate and except synchronous and metachronous multiple relative tumours (21.6 vs. $6 \%, P=0.001)$ and extracolorectal tumours (55.8 vs. $18.3 \%, P=0.000)$. These differences may be caused by gastric cancer patients in Fudan criteria families. In other words, patients who fulfilled Fudan criteria have similar clinicopathological features to the patients who fulfilled Amsterdam I/II criteria.

The contents of Fudan criteria were as followed: (1) There should at least three relatives with colorectal cancer or with an extracolonic cancer: cancer of endometrium, stomach, small bowel, ureter or renal pelvis. One relative should be a first-degree relative of the other two. (2) At least two successive generations should be affected. (3) At least one tumour should be diagnosed before age 50. (4) Familial adenomatous polyposis should be excluded. (5) Tumours should be verified by histopathological examination.

\section{Relationship between genotype and phenotype}

Approximately $85 \%$ of genetically defined HNPCC patients have germline mutations in MLH1 and MSH2 [3]. Several investigators have tried to correlate the phenotype with the affected gene [14]. Kastrinos et al. [37] reported that MLH1 carriers $(n=112)$ had a higher prevalence of colorectal cancer (79 vs. $69 \%, P=0.08$ ) and younger age of diagnosis (42.2 vs. 44.8 years, $P=0.03$ ) when compared to MSH2 carriers $(n=173)$. While the prevalence of endometrial cancer in women $(68 / 167,41 \%)$ was similar in both groups (36 vs. $44 \%$ ), other extracolonic cancers were more frequent in MSH2 carriers compared to MLH1 carriers (24 vs. $9 \%$; OR 3.2; $95 \%$ CI 1.5-6.6; $P=0.001$ ) and their families $(P<0.001)$. Choi et al. [38] analyzed 32 families from the Canadian familial colorectal cancer registry. Males with MLH1 mutations exhibited a significantly higher CRC risk than females (67 vs. $35 \%$ by age $70, P=0.02$ ), while the risk was similar in $\mathrm{MSH} 2$ carriers (about $54 \%$ ). The relative risk was constant with age (hazard ratio; between 5.5 and 5.1 over age 30-70) in MLH1 carriers, while the harzard ratio in MSH2 carriers decreased with age (from 13.1 at age 30 to 5.4 at age 70 ). In our study, families with hMSH2 mutation had a higher ratio of synchronous and metachronous colon cancers than families with hMLH1 mutation ( 33 vs. $6 \%, P=0.04$ ). So, we suggest patient with hMSH2 mutation need more 
frequent colonoscopy during follow-up. It has been recently reported that pathogenic PMS2 mutation is more frequently identified (4\%) than originally expected [39]. Hendriks et al. [40] reported that the cumulative risk for Lynch associated tumours was significantly lower in MSH6 carriers when compared to MLH1 or MSH2 mutation carriers $(P=0.002)$. Watson et al. [41] reported the risk of colorectal cancer in MSH6 carriers in the Dutch HNPCC database. The median age of onset of colorectal cancer in putative mutation carriers was 10 years higher for MSH6 (54 years; $95 \%$ CI 51-56) compared with MLH1 and MSH2 carriers (44 years; $95 \%$ CI 43-45). MSH6 families also showed a lower incidence of colorectal cancer compared with MLH1 and MSH2 families $(P<0.001)$.Therefore, in the future study on HNPCC, MSH6 and PMS2 should be considered to be included.

Acknowledgments We confirm that the article is our original work, it has not been published elsewhere and that it has not been submitted simultaneously for publication elsewhere.

Conflict of interest None declared.

Open Access This article is distributed under the terms of the Creative Commons Attribution License which permits any use, distribution, and reproduction in any medium, provided the original author(s) and the source are credited.

\section{References}

1. Giardiello FM, Brensinger JD, Petersen GM. AGA technical review on hereditary colorectal cancer and genetic testing. Gastroenterology. 2001;121(1):198-213.

2. Lynch HT, de la Chapelle A. Genetic susceptibility to non-polyposis colorectal cancer. J Med Genet. 1999;36(11):801-18.

3. Fishel R, Lescoe MK, Rao MR, et al. The human mutator gene homolog MSH2 and its association with hereditary nonpolyposis colon cancer. Cell. 1993;75(5):1027-38.

4. Bronner CE, Baker SM, Morrison PT, et al. Mutation in the DNA mismatch repair gene homologue hMLH1 is associated with hereditary non-polyposis colon cancer. Nature. 1994;368(6468): 258-61.

5. Nicolaides NC, Papadopoulos N, Liu B, et al. Mutations of two PMS homologues in hereditary nonpolyposis colon cancer. Nature. 1994;371(6492):75-80.

6. Miyaki M, Konishi M, Tanaka K, et al. Germline mutation of MSH6 as the cause of hereditary nonpolyposis colorectal cancer. Nat Genet. 1997;17(3):271-2.

7. Peltomaki P, Vasen H. Mutations associated with HNPCC predisposition-update of ICG-HNPCC/INSiGHT mutation database. Dis Markers. 2004;20(4-5):269-76.

8. Lynch HT, de la Chapelle A. Hereditary colorectal cancer. N Engl J Med. 2003;348(10):919-32.

9. Chen S, Wang W, Lee S, et al. Prediction of germline mutations and cancer risk in the Lynch syndrome. JAMA. 2006;296(12):1479-87.

10. Rodriguez-Bigas MA, Boland CR, Hamilton SR, et al. A National Cancer Institute workshop on hereditary nonpolyposis colorectal cancer syndrome: meeting highlights and Bethesda guidelines. J Natl Cancer Inst. 1997;89(23):1758-62.
11. Umar A, Boland CR, Terdiman JP, et al. Revised Bethesda Guidelines for hereditary nonpolyposis colorectal cancer (Lynch syndrome) and microsatellite instability. J Natl Cancer Inst. 2004;96(4):261-8.

12. Syngal S, Fox EA, Eng C, Kolodner RD, Garber JE. Sensitivity and specificity of clinical criteria for hereditary non-polyposis colorectal cancer associated mutations in MSH2 and MLH1. J Med Genet. 2000;37(9):641-5.

13. Wei W, Liu L, Chen J, et al. Racial differences in MLH1 and MSH2 mutation: an analysis of yellow race and white race based on the InSiGHT database. J Bioinform Comput Biol. 2010;8(Suppl 1): 111-25.

14. Chew MH, Koh PK, Ng KH, et al. Phenotypic characteristics of hereditary non-polyposis colorectal cancer by the Amsterdam criteria: an Asian perspective. Anz J Surg. 2008;78(7):556-60.

15. Cai SJ, Xu Y, Cai GX, et al. Clinical characteristics and diagnosis of patients with hereditary nonpolyposis colorectal cancer. World J Gastroenterol. 2003;9(2):284-7.

16. Goecke T, Schulmann K, Engel C, et al. Genotype-phenotype comparison of German MLH1 and MSH2 mutation carriers clinically affected with Lynch syndrome: a report by the German HNPCC Consortium. J Clin Oncol. 2006;24(26):4285-92.

17. Park YJ, Shin KH, Park JG. Risk of gastric cancer in hereditary nonpolyposis colorectal cancer in Korea. Clin Cancer Res. 2000;6(8):2994-8.

18. Zhang YZ, Sheng JQ, Li SR, et al. Clinical phenotype and prevalence of hereditary nonpolyposis colorectal cancer syndrome in Chinese population. World J Gastroenterol. 2005;11(10):1481-8.

19. Yoon S, Park TS, Kim NK, et al. Three new nonsense mutations of MLH1 and MSH2 genes in Korean families with hereditary nonpolyposis colorectal cancer. Cancer Genet Cytogenet. 2009;188(2): $61-4$.

20. Lynch HT, Boland CR, Gong G, et al. Phenotypic and genotypic heterogeneity in the Lynch syndrome: diagnostic, surveillance and management implications. Eur J Hum Genet. 2006;14(4): $390-402$.

21. Lu KH, Schorge JO, Rodabaugh KJ, et al. Prospective determination of prevalence of lynch syndrome in young women with endometrial cancer. J Clin Oncol. 2007;25(33):5158-64.

22. Myrhoj T, Bisgaard ML, Bernstein I, Svendsen LB, Sondergaard JO, Bulow S. Hereditary non-polyposis colorectal cancer: clinical features and survival. Results from the Danish HNPCC register. Scand J Gastroenterol. 1997;32(6):572-6.

23. Percesepe A, Benatti P, Roncucci L, et al. Survival analysis in families affected by hereditary non-polyposis colorectal cancer. Int J Cancer. 1997;71(3):373-6.

24. Lindgren G, Liljegren A, Jaramillo E, Rubio C, Lindblom A. Adenoma prevalence and cancer risk in familial non-polyposis colorectal cancer. Gut. 2002;50(2):228-34.

25. Goecke T, Schulmann K, Engel C, et al. Genotype-phenotype comparison of German MLH1 and MSH2 mutation carriers clinically affected with Lynch syndrome: a report by the German HNPCC Consortium. J Clin Oncol. 2006;24(26):4285-92.

26. Kim JC, Kim HC, Roh SA, et al. hMLH1 and hMSH2 mutations in families with familial clustering of gastric cancer and hereditary non-polyposis colorectal cancer. Cancer Detect Prev. 2001;25(6):503-10.

27. Oliveira FF, Napoli FC, Rossi BM, et al. Frequency of extracolonic tumors in hereditary nonpolyposis colorectal cancer (HNPCC) and familial colorectal cancer (FCC) Brazilian families: an analysis by a Brazilian Hereditary Colorectal Cancer Institutional Registry. Fam Cancer. 2004;3(1):41-7.

28. Chung DC, Rustgi AK. The hereditary nonpolyposis colorectal cancer syndrome: genetics and clinical implications. Ann Intern Med. 2003;138(7):560-70. 
29. Lynch HT, Smyrk TC, Watson P, et al. Genetics, natural history, tumor spectrum, and pathology of hereditary nonpolyposis colorectal cancer: an updated review. Gastroenterology. 1993;104(5): 1535-49.

30. Park JG, Park YJ, Wijnen JT, Vasen HF. Gene-environment interaction in hereditary nonpolyposis colorectal cancer with implications for diagnosis and genetic testing. Int $\mathrm{J}$ Cancer. 1999;82(4):516-9.

31. Fornasarig M, Campagnutta E, Talamini R, et al. Risk factors for endometrial cancer according to familial susceptibility. Int $\mathbf{J}$ Cancer. 1998;77(1):29-32.

32. Aarnio M, Salovaara R, Aaltonen LA, Mecklin JP, Jarvinen HJ. Features of gastric cancer in hereditary non-polyposis colorectal cancer syndrome. Int J Cancer. 1997;74(5):551-5.

33. Watson P, Lynch HT. Extracolonic cancer in hereditary nonpolyposis colorectal cancer. Cancer. 1993;71(3):677-85.

34. Vasen HF, Wijnen JT, Menko FH, et al. Cancer risk in families with hereditary nonpolyposis colorectal cancer diagnosed by mutation analysis. Gastroenterology. 1996;110(4):1020-7.

35. Aarnio M, Mecklin JP, Aaltonen LA, Nystrom-Lahti M, Jarvinen HJ. Life-time risk of different cancers in hereditary non-polyposis colorectal cancer (HNPCC) syndrome. Int J Cancer. 1995;64(6):430-3.
36. O'Leary TJ. Molecular diagnosis of hereditary nonpolyposis colorectal cancer. JAMA. 1999;282(3):281-2.

37. Kastrinos F, Stoffel EM, Balmana J, Steyerberg EW, Mercado R, Syngal S. Phenotype comparison of MLH1 and MSH2 mutation carriers in a cohort of 1,914 individuals undergoing clinical genetic testing in the United States. Cancer Epidemiol Biomarkers Prev. 2008;17(8):2044-51.

38. Choi YH, Cotterchio M, McKeown-Eyssen G, et al. Penetrance of colorectal cancer among MLH1/MSH2 carriers participating in the colorectal cancer familial registry in Ontario. Hered Cancer Clin Pract. 2009;7(1):14.

39. Niessen RC, Kleibeuker JH, Westers H, et al. PMS2 involvement in patients suspected of Lynch syndrome. Genes Chromosomes Cancer. 2009;48(4):322-9.

40. Hendriks YM, Wagner A, Morreau $\mathrm{H}$, et al. Cancer risk in hereditary nonpolyposis colorectal cancer due to MSH6 mutations: impact on counseling and surveillance. Gastroenterology. 2004;127(1):17-25.

41. Watson P, Vasen HF, Mecklin JP, et al. The risk of extra-colonic, extra-endometrial cancer in the Lynch syndrome. Int $\mathrm{J}$ Cancer. 2008;123(2):444-9. 